\title{
Research of the Thermal Parameters and the Accuracy of Flow Measurement of the Biological Fuel
}

\author{
Igor Korobiichuk ${ }^{1}$, Shavursky Yurij ${ }^{2}$, Michal Nowicki $^{3}$ and Roman Szewczyk ${ }^{3}$ \\ 1. Industrial Research Institute for Automations and Measurements PIAP, Warsaw 02-486, Poland \\ 2. Zhytomyr State Technological University, Zhytomyr 10005, Ukraine \\ 3. Institute of Metrology and Biomedical Engineering, Warsaw University of Technology, Warsaw 02-525, Poland
}

\begin{abstract}
The heat parameters, the thermoanemometric flow-meter (TAF) errors and the experimental characteristics have been defined. The results of experiments were conducted with the help of physically-informational models allowing to realize all major thermal methods and their inherent informative options. The metrological evaluation was made and the sensitivity to the consumption of gas and liquid have been defined, their static and dynamic errors, followed by the comparison of costs according to these criteria. The developed method provides accurate measurement of volumetric flow of motor fuel $1.0-1.5 \%$ at heater temperature measurement accuracy of $1 \%$.
\end{abstract}

Key words: Thermoanemometric flow-meter, biological fuel, physically-informational models.

\section{Introduction}

Despite the wide range of the flow-measuring tools, there exists such known as: ranges of small-and-microexpenses; and by their properties-complex, for example, multiphase flows [1]. For measuring of the biological fuel flow, the most present promising non-contact measurement means are used-ultrasonic, coriolis and the heat ones [2, 3]. But the existing thermal flowmeters (hot-wire and the calorimetric type) do not provide information about the biofuels flow in the stream and the area of their functioning is limited to small values. This work is devoted to the methods of the heat measurement, which possess a wide informative and high operational reliability, for the purpose of the modern computing devices utilization [4]. To solve the most tasks, it is urgent to use the combined thermal methods and creation of the new flow-meters structures allowing to do the flow fully automated and with the high speed [5].

Corresponding author: Igor Korobiichuk, Ph.D., research fields: measuring systems, and automation of control systems. E-mail: ikorobiichuk@piap.pl.

\section{Analysis of the Recent Studies}

For the exprimental research of the thermoanemometric flow-meter (TAF), the following methods associated with the speed measuring of the air flow, gas and mixtures of liquids were reviewed.

But the information about the study of the fuels-flow using rapeseed oil is practically absent [6-8].

The need for such research is due in that the physico-chemical properties of the biofuels significantly differ from those conventional motor fuels (petrol and diesel fuel). These significant differences influence the course of flow through biofuels flowmeter to the work of flowmeter dynamic mode and its mistakes.

Therefore, the experimental thermal TAF balance research was conducted. Seven options of the motor fuels costs considering the motor fuels temperature field errors and on the basis of approximation were studied. The accuracy of measurement using algorithmic procedures was improved.

The aim of this work is the development of the physical model of the primary measuring converter, the experimental research of the thermoanemometric 
biofuels flowmeter and testing the installation in the cars with the internal combustion diesel engine.

\section{The Presentation of the Main Material}

\subsection{The Method of the Biological Fuel Measurement}

The most actual method of the biological fuel measurement with the help of the contactless meter is presented in this article.

Among the known flowmeters the thermal measurement method was used and improved. Among them is the well-known in use thermoanemometric flow meter to measure the amount and rate of fuel in the automobile internal combustion engines [9].

The experimental unit, the procedure of study, three options of physical models, and the results of experiments were presented to confirm the effectiveness of the use of thermal methods for measurement of biofuels in the fuel flow through the conduit and of the properties of liquid phase, which were implemented in accordance with these structures. Fig. 1 illustrates the effect of liquid phase onto the temperature of boundary layer for different mixture flow regimes and confirms the application of the thermal layer method for the measurement of different fuels flow.

The main volume of experiments was conducted with the help of physically-informational models allowing to realize all major thermal methods and their inherent informative options.

The metrological evaluation was made and the sensitivity to the consumption of gas and liquid have been defined, their static and dynamic errors, followed by the comparison of costs according to these criteria.

\subsection{The Experimental Equipment}

Before the conduction of this experiment, we used the thermoanemometric flowmeter designed as two pipelines, one of which was connected from the fuel tank to the internal combustion engine, and the other conduit vice versa.

The greatest use among the modern devices to measure the temperature found the thermoelectric and

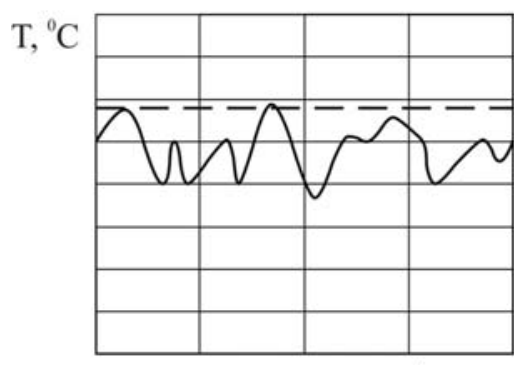

time, $s$

(a)

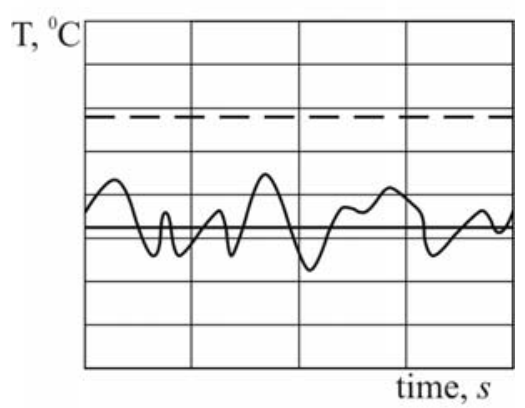

(b)

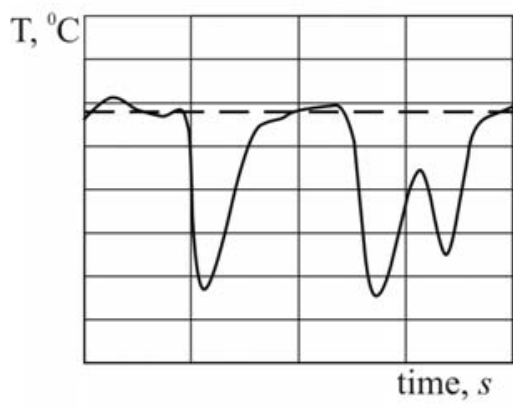

(c)

Fig. 1 (a) The mode using the diesel fuel with the rapeseed oil content; (b) the mode using petrol; and (c) the mode using diesel fuel. Cross-hatching line shows the initial temperature to a liquid state of fuels.

the thermistor sensors of temperature because of their simplicity, stability, performance and the temperature conversion capabilities directly into the electrical value.

For the better heat transfer between the walls of the shell and nickel wires are the conductive silver plates, insulated from the wire by thin mica gaskets. The body of sensor has a thickened head with a thread, designed for mounting of the sensor.

In the sensor head, there is a connector and managing additional resistance, connected in series with nickel winding. 
Additional resistance is designed to bring the temperature coefficient of the sensor to its default value to ensure the mutual replacement of sensors. Further was held (TAF) settings and the adjustment of its electrical elements.

This device (TAF) was used in the internal combustion diesel engine using diesel fuel and rapeseed oil (Fig. 2). To do this, it is necessary to combine the elements of engine with TAF.

We fix the TAF with the help of a harness in the housing of engine compartment, and isolate all the electrical components, to avoid short circuit.

In the wiring diagram (Fig. 3), there are two resistors of adjustment ( $R 3$ and $R 6$ ), with the help of one of which $(R 3)$, we can set the temperature onto $0{ }^{\circ} \mathrm{C}$.

Without changing the exponents of all the other thermal converters relative to zero, we proceed to the correction of the second resistor (R6), to adjust the temperature up to $100{ }^{\circ} \mathrm{C}$.

In order to make sure that all the sensors are configured in synchronization, we use an oscilloscope for the detention of all the indicators within the same band.

The use of such a method allows to identify the possible measurement error with changes in the temperature range of $0-180{ }^{\circ} \mathrm{C}$. The error is $0.2 \%$.
Fig. 4 graphically depicts modeling of the measuring errors of the volumetric flow rate of motor fuel, which were run with the help of the MATLAB program, depending on the temperature measurement errors using the thermocouples.

Further information on the errors modeling was passed onto the aggregate TAF device where it was further processed by a microprocessor [10]. The range of relative thermocouple errors was considered as from $0.01 \%$ to $1 \%$.

Modeling of the flow-meter errors was performed using three types of motor fuels (gasoline, diesel fuel and biodiesel).

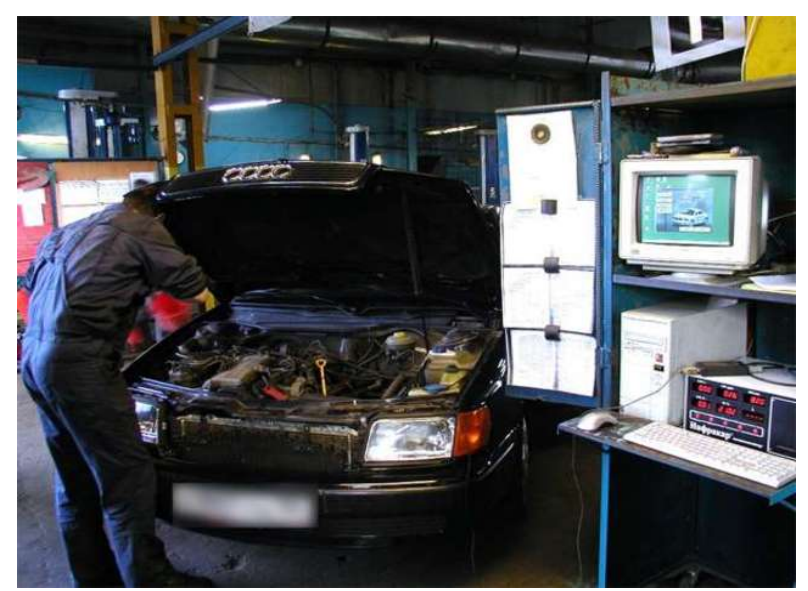

Fig. 2 A car with a diesel internal combustion engine.

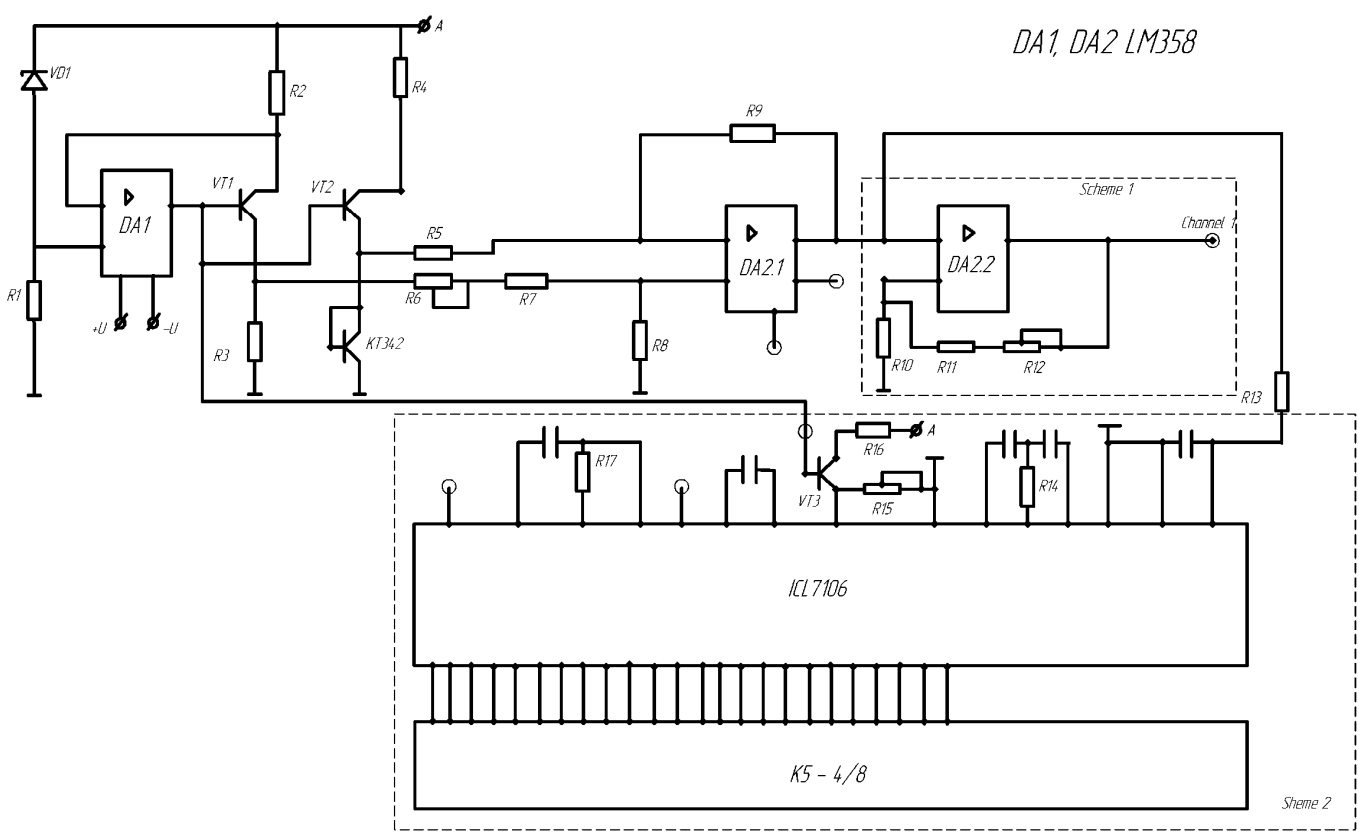

Fig. 3 Schematic diagram of the TAF complex. 


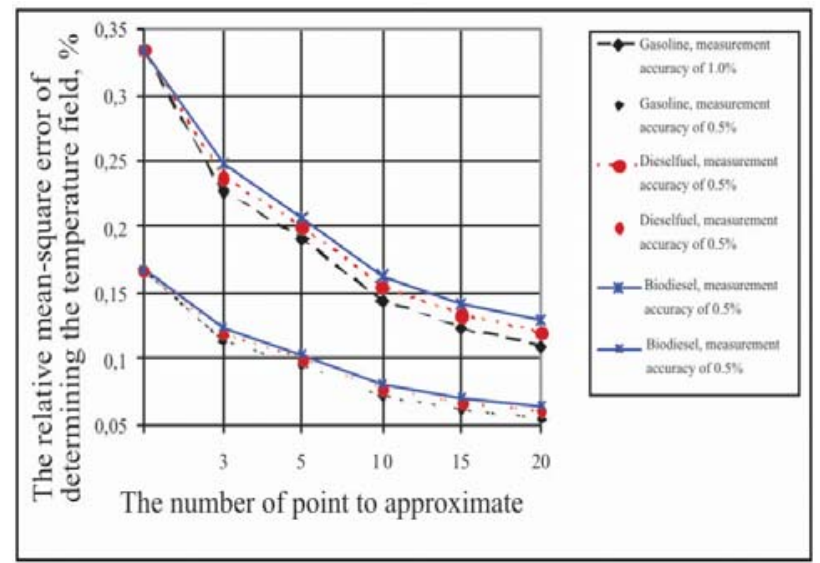

Fig. 4 The relative mean-square errors of determining the temperature field in a moving stream of motor fuels on the basis of approximation (thermocouples temperature measurement accuracy of $1.0 \%$ and $0.5 \%$ ).

\subsection{The Options for the Computer Determining Cost of Motor Fuel}

In this publication, seven options for the computer determining cost of motor fuels are being considered:

(1) Determining of the costs basing on the measurement of heater temperature and the initial temperature of motor fuel

$$
W_{M \Pi}=K_{4} \cdot K_{5} \cdot\left(T_{M \Pi O}-T_{H}\right)^{-\frac{1}{K_{2}}}
$$

where, $K_{4}=\frac{\pi d_{m p}}{4} \cdot\left(\frac{P_{\partial \varkappa}}{\pi \ell_{H}}\right)^{\frac{1}{K_{2}}}$ is the coefficient, which takes into account the design parameters of flow-meter, $K_{\sigma}=v_{M I} \cdot\left(\mu_{M I} \cdot C_{M I I}\right)^{-\frac{K_{3}}{K_{2}}} \cdot\left(\lambda_{M I I}\right)^{\frac{K_{3}-1}{K_{2}}} \cdot\left(K_{1}\right)^{-\frac{1}{K_{2}}}$ is the coefficient, that takes into account physical and chemical properties of motor fuel and the mode of its current flow through the flow-meter;

(2) Determining the outlay based on the measurement of motor fuels initial temperature, and motor fuel temperature at two points along the tube axis with averaging the results for these two points

$$
W_{M \Pi}=\left(\frac{K_{8} \cdot K_{9} \cdot x}{\ln \left[T(x)-T_{o m}\right]-\ln \left[T_{H}-T_{o m}\right]}\right)^{5}
$$

where, $K_{8}=-0.11 \cdot\left(\frac{\pi}{4}\right)^{0,2}, K_{9}=\frac{\left(v_{M I}\right)^{0,2}}{\left(d_{m p}\right)^{0,8}}$;
(3) Improving the accuracy of variant 2 based on the algorithmic compensation of random and dynamic errors using artificial neural networks [4];

(4) Improving the accuracy of variant 1 based on the approximation of the temperature measurement results using thermocouples and on the compensation of measurement errors;

(5) Improving the accuracy of variant 2 based on the approximation of the temperature measurement results using thermocouples and on the compensation of measurement errors;

(6) Improving the accuracy of variant 1 based on:

- algorithmic compensation of random and dynamic errors using artificial neural networks (according to the patent);

- approximation of the temperature measurement results by the thermocouples and of the compensation of these measurement errors;

(7) Improving the accuracy of variant 2 based on:

- algorithmic compensation of random and dynamic errors using artificial neural networks (according to the patent);

- approximation of the temperature measurement results by the thermocouples and of the compensation of these measurement errors according to the patent.

\section{Conclusions}

With the minimal amounts of algorithmic computation, the most appropriate is to use option 1 (the determining of outflow is based on measuring the temperature of the heater and the initial temperature of the motor fuel in accordance with the formula 1). This approach provides accurate measurement of volumetric flow of motor fuel $1.0-1.5 \%$ at heater temperature measurement accuracy of $1 \%$.

To improve the accuracy of flow-meter, it is necessary to use more complex algorithmic procedures (approximation of measurement results based on the method of the least squares and using artificial neural networks). To speed up the flow-meter in this case, it is necessary to include into the structure of the digital 
computer the specialized neural processor that realizes compensation of random and dynamic measurement error based on the theory of artificial neural networks. This approach provides accurate measurement of volumetric flow of motor fuel $1.0-1.5 \%$ at the heater temperature measurement accuracy of $1 \%$.

\section{References}

[1] Aheykyn D. I. 1985. Sensor control and regulation / D.I. Aheykyn, E.N. Kostin, N.N. Kuznetsova. - M: Mashinostroenie, 1985. - 928 p.

[2] Garrick, R. D., Villasmil, L., Lee, J., Gutterman, J. S. 2011. "Comparison of New Ultrasonic and Hot Wire Thermo-Anemometer Gas Flow Meters." In Proceedings of FIIW (Future of Instrumentation International Workshop), 164-7.

[3] Frenzel, F., Grothey, H., Habersetzer, C., Hiatt, M. 2011. Industrial Flow Measurement Basics and Practice. Germany: ABB Automation Products GmbH.

[4] Biryukov B. V. 1975. "The Functions of Influence of the Operating Conditions of the Systems of Accurate Reproduction and Measuring of the Fluid Flow." Measuring Equipment 4: 69-72.
[5] Pat. 91160 C2 Ukraine, IPC (2009) G 01 F 1/68. Highly Precise Motor Fuel Flow-Meter with the Digital Processing of Measurement Data/Bezvesilna, O. M., Podchashynsky Y.O. Ilchenko A.V., Shavursky Y.O.; the applicant and patentee - Zhytomyr State Technological University. - № a2009 06515; appl. 22.06.09; publ. 25.06.10, Bul. Number 12.

[6] Xu, L. J., Han, J., Wang, Y. 2005. "Design of Electrode Array of Inductance Flowmeter." IEEE Sensors Journal 5 (5): 929-33.

[7] Fei, L. Z., Zheng, D., Kai, Z., Sheng, L. D., Di, S. Z., Wei, Y. W. 2013. "Design and Analysis of Flow Rectifier of Gas Turbine Flowmeter." Thermal Science 17 (5): 1504-7.

[8] Filippov, A., Mjagkov, V. I., Atamanenko, M. E., and Topchiy, S. I. 2008. "Thermoanemometer Air Flow-Meter of the Internal Combustion Engine." Scientific Reports of NAU 3 (11): 21-7.

[9] Bezvesilna O., Tkachuk A., Korobiichuk I., Shadura V., Nowicki M., and Szewczyk R. 2015. "The Mathematical Model of Thermo-Anemometric Flowmeter.” Presented at the International Conference Mechatronics Ideal for Industrial Application, Gdańsk, Poland.

[10] Sergienko A. B. 2003. Digital Signal Processing. - SPb. Piter -608 p. 